\title{
A novel BSD domain-containing transcription factor controlling vegetative growth, leaf senescence and fruit quality in tomato
}

\author{
YOUHONG FAN ${ }^{1}$, Xiangli Niu ${ }^{1}$, Li Huang ${ }^{2}$, Rachel Gross ${ }^{2}, \mathrm{Han} \mathrm{Lu}^{1}$, Min Miao ${ }^{1}$, \\ Yongsheng $\mathrm{Liu}^{1}$, and Fangming $\mathrm{XiaO}^{2}$ \\ ${ }^{1}$ Hefei University of Technology \\ ${ }^{2}$ University of Idaho
}

April 28, 2020

\begin{abstract}
BSD (mammalian BTF2-like transcription factors, Drosophila synapse-associated proteins and yeast DOS2-like proteins) is a domain existing in a variety of organisms but its function has not been well studied. In this study, we identified a novel BSD domain-containing protein (SIBSD1) in tomato. Biochemical and subcellular assay indicated SIBSD1 is a functional transcription factor and predominantly localized in the nucleus. The genetic analyses suggested that SIBSD1 is a novel negative regulator of vegetative growth and leaf senescence in tomato. The SIBSD1-knockdown tomato plants exhibited retarded vegetative growth and precocious leaf senescence, whereas SIBSD1-overexpression tomato plants displayed the opposite phenotypes. The negative role of SIBSD1 in leaf senescence was also supported by RNA-Seq analysis on the SIBSD1-knockdown tomato leaf in comparison with the wild type tomato leaf. Moreover, altered soluble solids contents in fruits were detected in the SIBSD1-knockdown and SIBSD1-overexpression tomato plants. Taken together, our data suggested that the novel transcription factor SlBSD1 plays important roles in controlling fruit quality and other physiological processes in tomato, including vegetative growth and leaf senescence.
\end{abstract}

\section{Key words}

leaf senescence, SIBSD1, tomato, transcription factor, vegetative growth

\section{Introduction}

Given the fact that massive reprogramming of gene expression is required during plant development and growth, many transcription factors act as key regulators due to their potential activation or repression on many genes' expression cooperatively. Thus, identification and characterization of novel transcription factors would help us better understand the complicated processes of plant growth and development. Nearly two decades ago, a novel domain, BSD (mammalian $B$ TF2-like transcription factors, Drosophila $s$ ynapseassociated proteins and yeast $D$ OS2-like proteins), was found present in basal transcription factors, synapseassociated proteins and several hypothetical proteins (Doerks, Huber, Buchner, \& Bork, 2002). The BSD domain is featured with three $\alpha$-helices likely involved in DNA binding, with two highly conserved adjacent tryptophan and phenylalanine residues located at the C-terminus (Doerks et al., 2002). Limited studies have suggested BSD-containing proteins are involved in diverse physiological processes in a variety of species ranging from primal protozoan to human. For example, yeast DELOCALIZATION OF SWI6 (DOS2) plays an important role in heterochromatic histone modification and RNA interference (Li et al., 2005); drosophila SYNAPSE-ASSOCIATED PROTEIN OF 47 KDA (SAP47) is required for association function and shortterm plasticity of synapses (Reichmuth et al., 1995); mammalian BTF2H1 and its yeast homologue TFB1 are the component of the general transcription and DNA repair factor IIH core complex, which play a role in transcription initiation and nucleotide excision repair of damaged DNA (Iben et al., 2002; Wang et 
al., 1995). In plants, BSD-containing transcription factors have been identified in Arabidopsis and banana (Musa acuminata) (Ba et al., 2014; Park, Kim, Jung, \& Suh, 2009). However, the functionality of such BSD-containing transcription factors in plants has not been well studied, despite that it has been speculated the BSD-containing transcription factor plays a role in cell proliferation during somatic embryogenesis and is involved in ethylene-mediated fruit ripening in banana (Ba et al., 2014).

Leaf senescence, as the last stage of leaf development, is a highly regulated developmental process associated with the degradation of chlorophyll and macromolecules, subsequent mobilization of nutrients to actively growing organs (new buds, young leaves, developing seeds and fruits), and eventually massive programmed cell death (Lim, Kim, \& Nam, 2007). Senescence of leaves occurs in an age-dependent manner under optimal conditions, whereas when plants are exposed to nutrient deficiency or environmental stresses, leaf senescence can be induced precociously as an adaptive response to promote survival and reproduction (Guo \& Gan, 2005; Woo, Kim, Lim, \& Nam, 2019). Global transcriptome analysis has revealed that reprogramming of gene expression occurs during developmental or dark-induced leaf senescence in various plant species, including Arabidopsis (Breeze et al., 2011; V. Buchanan-Wollaston et al., 2005), wheat (Gregersen \& Holm, 2007), maize (Zhang et al., 2014) and aspen (Andersson et al., 2004). In general, in a senescing leaf, genes essential for chloroplast activity, including those involved in photosystems, carbon fixation, chlorophyll biosynthesis and amino acid metabolism, are down-regulated, while a subset of genes, mainly involved in chlorophyll and macromolecule catabolism and generally referred to as senescence-associated genes (SAGs), are up-regulated (Woo et al., 2019).

Transcription factors have been demonstrated to act as key regulators of senescence by activating or repressing senescence-related genes' expression cooperatively. In Arabidopsis, NAM/ATAF/CUC (NAC) is the major transcription factor family controlling leaf senescence. At least four NAC transcription factors, ANAC092/ORESARA1 (ORE1) (J. H. Kim et al., 2009), ANAC029/Arabidopsis NAC-LIKE Activated by AP3/PI (AtNAP) (Guo \& Gan, 2006), ANAC059/ORESARA1 SISTER1 (ORS1) (Salma Balazadeh et al., 2011) and ANAC016 (Y. S. Kim, Sakuraba, Han, Yoo, \& Paek, 2013), act as positive regulator of leaf senescence, whereas two NAC family members, ANAC042/JUNGBRUNNEN1 (JUB1) (Wu et al., 2012) and ANAC083/VND-INTERACTING2 (VNI2) (Yang, Seo, Yoon, \& Park, 2011), function as negative regulator. Among them, ORE1 is a master positive regulator controlling the expression of numerous SAGs by directly binding to their promoters (S. Balazadeh et al., 2010; Matallana-Ramirez et al., 2013). In tomato (Solanum lycopersicum ), the orthologs of ORE1 (SlORE1S02, SlORE1S03, and SlORE1S06) positively regulate leaf senescence (Lira et al., 2017); Two additional NAC transcription factors, NOR and SlNAP2, act as a positive regulator module for leaf senescence: SINAP2 activates NOR expression by directly binding to its promoter and, together with NOR, it jointly regulates two senescence-related genes SlSAG113 and SlSGR1 ; and SINAP2 also directly regulates at least four other senescence-related genes,SlSAG15 , SlPPH , SlKFB20 , and SlYLS4 (Ma, Balazadeh, \& Mueller-Roeber, 2019; Ma et al., 2018).

In this study, we identified a novel BSD-containing protein termed SIBSD1 in tomato that plays significant roles in diverse aspects of plant development and growth. SIBSD1 is a functional transcription factor and predominantly localized in the nucleus. Significantly, SIBSD1 negatively regulates vegetative growth and senescence, as manifested by opposite pleiotropic phenotypes in transgenic tomato plants with knockdown or overexpression of SIBSD1. These phenotypes included retarded or promoted vegetative growth, precocious or delayed leaf senescence, and altered soluble solids content in fruits.

\section{Materials and Methods}

\section{Plant material and growth conditions}

Tomato (Solanum lycopersicu m cv. Ailsa Craig), obtained from the C. M. Rick Tomato Genetics Resource Center (University of California, Davis, USA) and Nicotiana benthamiana were grown under natural light

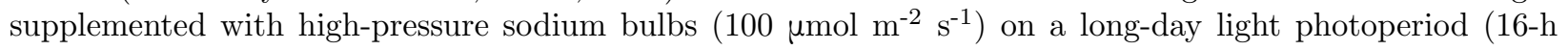
light/8-h dark). Daytime and nighttime temperatures were 24 to 28 and 16 to 20 , respectively. The relative humidity was $50 \%$ to $70 \%$. 


\section{Generation of transgenic tomato plants}

The binary vectors pBI121 were transformed into tomato by Agrobacterium tumefaciens-mediated transformation (Van Eck, Kirk, \& Walmsley, 2006). After in vitro regeneration of transgenic plants, well-rooted plants were transplant into moistened soil in plastic pots. Plants were covered with transparent plastic cups and maintained in a shaded area for acclimatization. 1 week later, the plants were moved to greenhouse and the plastic cups were removed.

\section{Yeast two-hybrid and transcription activation assays}

The LexA-based yeast two-hybrid $(\mathrm{Y} 2 \mathrm{H})$ system was used to determine the interactions between SISINA1 and SIBSD1. The full-length cDNAs ofSIBSD1 and SISINA1 genes were cloned into the bait vector pEG202 and the prey vector pJG4-5, respectively. The yeast (Saccharomyces cerevisiae) strain EGY48 harboring the LacZmark gene was transformed with the bait and prey constructs in the appropriate combinations. The transformed yeast cells were streaked onto X-Gal plates to assess the interaction between SlBSD1 and SISINA1. For transcription activation assays, the full-length cDNA of SlBSD1 was cloned into pEG202 in frame with the LexA-coding region. The construct was introduced into yeast stain EGY48 containing the lacZ reporter plasmid pSH18-34 to test the possible activation of the reporter genes. Photographs were taken after incubation at $30^{\circ} \mathrm{C}$ for 2 days.

\section{Transient expression experiments}

Agrobacterium-mediated transient expression on $N$. benthamianaleaves was performed as described previously (Sessa, D'Ascenzo, \& Martin, 2000). A. tumefaciens cells carrying appropriate constructs were syringeinfiltrated into $N$. benthamiana leaves. Agroinfiltrated leaf tissues were collected $36 \mathrm{~h}$ after agroinfiltration. Proteins were extracted from leaf tissues or protoplasts, resolved by SDS-PAGE and analyzed by immunoblotting. All transient expression experiments were repeated at least three times with similar results.

\section{Total chlorophyll content measurement}

Leaves was extracted with $80 \%$ acetone at 4 for $14 \mathrm{~h}$ in darkness. Total chlorophyll per fresh weight of leaves was calculated as described previously (Arnon, 1949).

\section{Trypan blue staining}

The trypan blue staining assays were conducted as described (Fernandez-Bautista, Dominguez-Nunez, Moreno, \& Berrocal-Lobo, 2016) with minor modifications. Leaves were detached and submerged in $0.2 \%$ lactophenol trypan blue solution ( $0.2 \%$ trypan blue, $25 \%$ lactic acid, $25 \%$ water-saturated phenol, and $25 \%$ glycerol). The samples were washed in $95 \%$ ethanol to reduce background staining.

\section{RT-qPCR analysis}

Total RNA was isolated from leaf tissues using TRIzol reagent (Ambion, Carlsbad, CA, USA) and cDNA was synthesized using SuperScript IV First-Strand Synthesis System (Thermo Fisher Scientific, Vilnius, Lithuania). Real-time PCR was performed using SYBR Green (Life Technologies, Warrington, UK) and an ABI 7300 Real-time PCR system (Applied Biosystems, Foster City, CA, USA). Actin-41 (Solyc04g011500 ) served as reference gene for data analysis. Statistical significance was determined using student's $t$-test. Primers used for in RT-qPCR are listed in Table S1.

\section{RNA-Seq analyses}

RNA was extracted from SlBSD1-KD, SISINA1-OX and WT leaves using SPLIT RNA Extraction Kit (Lexogen, Greenland, NH, USA) following the manufacturer instructions. RNA samples were quantified and quality-checked on a Fragment Analyzer Automated CE System (Advanced Analytical, Ankeny, IA, USA). Libraries for sequencing were prepared using mRNA-Seq Library Prep Kit for Illumina (Lexogen, Greenland, NH, USA) following the manufacturer instructions. Libraries were sequenced at the University of Oregon on a SE100 run using an Illumina HiSeq 4000 platform. Approximately 5 million reads were generated for each sample library. High-quality trimmed reads were mapped to the genome of S. lycopersicum (genome version 
SL4.0 and annotation ITAG4.0) (Tomato Genome Consortium, 2012) and quantified using Salmon (Soneson, Love, \& Robinson, 2016). Differential expression analysis was then carried out using DESeq2 (Love, Huber, \& Anders, 2014).

\section{Accession Numbers}

Sequence data from key genes in this article can be found in the GenBank/EMBL/Solgenomics databases under the following accession numbers: SlBSD1 , Solyc04g077600 ; SlSAG12 ,Solyc02g076910 ; SlSAG15 , Solyc03g117950;SlSGR1, Solyc08g080090 ; SlPPH ,Solyc01g088090 .

\section{Results}

\section{Phylogenetic analysis of BSD domain-containing proteins}

A novel BSD domain-containing protein family, existing in a variety of organisms ranging from protozoans to humans, was newly identified by computational analysis (Doerks et al., 2002). In order to investigate the BSD domains found in plant proteins, we retrieved the amino acid sequences of BSD domains from 9 representative species (http://www.ebi.ac.uk/interpro/), including 3 plant species and 6 non-plant species. Highly similar sequences in the BSD domain were not included (for example the majority of BSD domains of human and mouse proteins are identical) and the remaining 63 unique sequences were used for multiple sequence alignment and phylogenetic analyses. This collection contained 16 unique BSD domain sequences in Arabidopsis, 12 in rice, 12 in tomato and 23 in other species. The multiple sequence alignment indicated that BSD domains are evolutionarily conserved and two less common amino acids, Phe and Trp, are adjacent and immutable (Fig. S1 ).

We utilized the Neighbor-Joining (NJ) method to construct a phylogenetic tree with 5 main clades, supported by high bootstrap values (Fig.1a ). Clade I and II were named the BTF2-like transcription factors, as they included BSD domain a and $\mathrm{b}$ in the well-studied BTF2H1/TFB1 transcription factors. Mammalian BTF2H1 and its yeast homolog TFB1 are the component of the general transcription and DNA repair factor IIH core complex, which plays a role in transcription initiation and nucleotide excision repair of damaged DNA (Iben et al., 2002; Wang et al., 1995). The third clade included BSD domain from Drosophila SAP47 was designated as the synapse-associated protein clade, despite many BSD domains in this Clade are also found in plant proteins without synapse activity. Clade IV, the DOS2-like clade, included BSD domain from yeast DOS2 which is required for heterochromatic histone modification and RNA interference (Li et al., 2005). Clade V contained BSD domains of plant proteins, including the BSD domain of the Arabidopsis BSD1 protein that has been characterized as a transcription activator (Park et al., 2009), suggesting this clade may evolve later with plant-specific functions.

We selected the tomato gene Solyc04g077600 for further analysis. The phylogenetic analysis revealed that Solyc04g077600 and Soly07g022920 are orthologs of AtBSD1 (Fig. 1b ). The Soly04g077600 contained a central BSD domain and was designated as SlBSD1 (Fig. 1c ), as it is the closest ortholog to AtBSD1. Although SlBSD1 and Soly07g022920 were paralogs, they were related to each other distantly ( $\sim 50 \%$ identity). Moreover, most BSD proteins, including SlBSD1 and Soly07g022920 protein, cluster in pairs (Figure 1b ), suggesting genome duplications for possible neofunctionalization.

\section{SIBSD1 is a functional transcription factor}

The presence of BSD domain in BTF2H1/TFB1 (a transcription factor and DNA repair factor subunit) and DOS2 (a heterochromatic histone modification-related protein) suggest that the BSD domain could have a role in chromatin-associated processes, which take place in the nucleus (Iben et al., 2002; Li et al., 2005; Wang et al., 1995). To analyze the subcellular localization of SlBSD1 in plant cells, a fusion construct of SlBSD1 fused with GREEN FLUORESCENCE PROTEIN (GFP) driven by the CaMV 35S promoter was generated and transiently expressed in $N$. benthamiana protoplasts, followed by the fluorescent microscopy. As shown in Fig. 2a , the fluorescence signal of free GFP protein was distributed evenly in both cytoplasm and nucleus, whereas the vast majority of SlBSD1-GFP-derived fluorescence signal was observed in the nucleus, suggesting SIBSD1 protein is predominantly localized in the nucleus. 
The identification as a transcriptional activator of AtBSD1 (Park et al., 2009), the Arabidopsis ortholog of SIBSD1, suggests SIBSD1 may possess potential transcriptional activity as well. To test this notion, a trans-activation assay was conducted in yeast. Expression of the LexA-DBD (DNA binding domain of the LexA operator) construct alone in yeast did not activate the lac $Z$ marker gene. In contrast, expression of the LexA-DBD-SIBSD1 fusion construct rendered the activation of the lac $Z$ gene, as manifested by the betagalactosidase activity of yeast cells grown on the X-Gal plate (Fig. 2b ), suggesting SlBSD1 is a functional transcriptional activator.

Transcription factors with distinct functions are often expressed in a tissue-specific manner, stimulating or depressing the transcription of associated genes in specific cell types. To determine whether the expression profiling of SlBSD1 is tissue-specific in tomato, we investigated the expression of SlBSD1 in different tissues. qRT-PCR analysis indicated SIBSD1 is expressed in all tested tissues (root, stem, leaf, bud, flower and fruit), with relatively lower expression level in leaves (Fig. 2c ).

\section{SIBSD1 is essential for vegetative growth}

To determine the physiological function of SIBSD1 in tomato, a loss-of-function approach was implemented using the RNA interference technique. Transgenic tomato lines with SlBSD1 knockdown were generated, of which two lines (SlBSD1-KD1 and SlBSD1-KD2) with most reduced SlBSD1 mRNA levels (70\% and 85\% reduction inSlBSD1-KD1 and SlBSD1-KD2 respectively, Fig. S2a ) were selected for further analysis. The mRNA level of Soly07g022920, the ortholog of SlBSD1, was unaffected in SlBSD1-KD1 orSlBSD1-KD2 (no statistically significant change, Fig.S2b ), indicating the specific silencing of the SIBSD1 target gene. One dramatic phenotypic change we observed was the vegetative growth of SlBSD1-KD plants is significantly affected. We examined the height of SlBSD1-KD plants in comparison with the wild type (WT) tomato plants through the growth at 4-week, 6-week and 13-week stage. The height of SlBSD1-KD plants was reduced $40.80 \%, 44.24 \%$ and $21.98 \%$ compared to that of WT plants, respectively, indicating knockdown of the SlBSD1 gene results in significant growth retardation in tomato (Fig. 3 ).

Next, we sought to verify the function of SIBSD1 in growth by the gain-of-function approach, via generating transgenic tomato constitutively over-expressing SlBSD1 under the control of the CaMV 35S promoter. Two transgenic lines with highest SlBSD1 expression (29- and 40-fold overexpression in SlBSD1-OX1 andSIBSD1OX2 respectively, Fig. S2c ) were selected for analysis. We found SlBSD1-OX plants display opposite vegetative growth phenotype as SlBSD1-KD plants did: at 4-week, 6-week and 13-week growth stages, SlBSD1-KD plants were $38.79 \%, 25.15 \%$ and $10.84 \%$ taller the WT plants at the same age, respectively (Fig.3 ). Thus, we concluded that SlBSD1 plays a positive role in vegetative growth in tomato.

\section{SIBSD1 negative regulates leaf senescence in tomato}

SlBSD1-KD plants did not exhibit any other morphological alterations except retarded vegetative growth at the early developmental stages (8-week-old). However, knockdown of SlBSD1 caused yellowing of mature green leaves in the 8-week-old SlBSD1-KDtomato plants, whereas the WT tomato leaves were still green at the same growth stage (Fig. 4a). Since leaf yellowing due to the preferential degradation of chlorophyll over carotenoids is the most obvious sign of senescence (Vicky Buchanan-Wollaston, 1997), we examined the senescence-associated characteristics of leaves at different growth ages in SIBSD1-KD tomato plants. Accelerated loss of chlorophyll content in the leaves at a later age $\left(7^{\text {th }}\right.$ and $4^{\text {th }}$ true leaves) was observed in SlBSD1-KD plants (Fig. 4b ). In addition, senescence-associated cell death was also enhanced in SlBSD1-KD plants, as shown by local patches of trypan blue-stained early-dying cells in the aged leaflets $\left(7^{\text {th }}\right.$ and $4^{\text {th }}$ true leaves, Fig.4c ).

To further elucidate the regulation of the early senescence phenotype of the SlBSD1-KD plants at the transcription level, we examined the expression of SAGs in these plants in comparison with the WT plants. Four senescence-related genes were examined. SlSAG12(Solyc02g076910, encoding a cysteine protease), SlSAG15 (Solyc03g117950, encoding a Clp ATPase), SlSGR1 (Solyc08g080090, encoding a stay-green protein involved in chlorophyll degradation) and SlPPH (Solyc01g088090, encoding a pheophytinase involved in chlorophyll degradation were induced during developmental senescence in the WT tomato leaves (Fig.S3a-d 
). Significantly, these 4 genes were significantly up-regulated in the $3^{\text {rd }}$ true leaf of 8-week-oldSlBSD1-KD plants compared to the WT leaves (Fig. 4d ), suggesting knockdown of SlBSD1 leads to precocious leaf senescence in tomato.

There was no significant difference in leaf senescence between the 8-week-old SlBSD1-OX and the WT plants (Fig. S4 ). However, at the 12-week-old age, the aged leaves of WT tomato plants began turning yellow, whereas the leaves of the same age ofSIBSD1-OX plants stayed green (Figure 5a ). Moreover, analyses of senescence-associated characteristics in the 12-week-oldSIBSD1-OX and the WT plants indicated that leaf senescence was significantly delayed in SlBSD1-OX plants, as shown by delayed loss of chlorophyll content (Fig. 5b ), the absence of trypan blue-stained dying cells (Fig. 5c ), and the delayed induction of senescence marker genes (Fig. 5d ). Taken together, these results suggest that constitutive over-expression of $S l B S D 1$ renders delayed leaf senescence in tomato. Thus, based on our loss-of-function and gain-of-function analyses, we conclude that SlBSD1 functions as a negative regulator of leaf senescence in tomato.

\section{SIBSD1 is involved in dark-induced leaf senescence}

Leaf senescence occurs in an age-dependent manner but is also regulated by a variety of endogenous and exogenous factors. Darkness is often considered as an exogenous inducer of leaf senescence and has been commonly used to induce synchronous senescence in detached leaves (Nooden, 1988). Although the darkinduced senescence in detached leaves shares many common features with developmental senescence, the underlying molecular mechanisms might not be identical. In fact, incubation of detached leaves in darkness induce overlapping but different sets of SAGs compared to developmental senescence (Quirino, Noh, Himelblau, \& Amasino, 2000). To test whether SIBSD1 is also involved in dark-induced leaf senescence, mature green leaflets from 6-week-old plants were incubated in the dark for up to 10 days. Our results indicate that, in comparison to those of the WT plants, detached leaflets of SlBSD1-KD plants showed enhanced yellowing under dark conditions, while leaflets detached from SlBSD1-OX plants remained relatively green (Fig. 6a,c ); the senescence-associated cell death was accelerated in SlBSD1-KDleaves but delayed in $S l B S D 1-O X$ leaves (Fig. 6b ); expression of senescence marker genes in $S l B S D 1-K D$ leaves was up-regulated but down-regulated in SlBSD1-OX leaves (Fig.6d ). Taken together, our data suggest SlBSD1 is involved in both developmental and dark-induced leaf senescence.

\section{RNA-seq analysis on the SIBSD1-KD and WT leaves}

To further investigate the molecular basis of SlBSD1-mediated regulation of leaf senescence, we sought to identify genes that are regulated by SIBSD1 during leaf senescence by RNA-seq analysis. To this end, we examined the transcriptomes of $S I B S D 1-K D$ leaves with those of WT leaves to determine differentially expressed genes when the expression of SlBSD1 is repressed, particularly genes involved in leaf senescence. As shown in Table 1 and Table S2, genes involved in degradation of macromolecules and mobilization were up-regulated in $S I B S D 1-K D$ leaves. In particular, increased transcript level was detected in genes encoding different types of proteases, including cysteine and aspartyl proteases, and genes encoding components of ubiquitin-proteasome pathway (such as ubiquitin-conjugating enzyme, F-box protein and proteasome subunit). In addition, genes encoding ribonuclease, which is involved in RNA degradation, were up-regulated, and one gene encoding chlorophyllase, a key enzyme in chlorophyll catabolic process, was up-regulated in SIBSD1-KD leaves as well. Moreover, many transporter genes exhibited increased transcript levels in SlBSD1-KD leaves, including genes coding for amino acid, peptide, sugar and cation transporters, indicating increased mobilization of nutrients from the senescing leaves to other parts of plants. On the other hand, many genes essential for chloroplast activity were down-regulated in SlBSD1-KD leaves, including genes encoding chlorophyll binding protein, photosystem I and II subunit, as well as genes involved in chlorophyll (tetrapyrrole) biosynthetic process. In addition, genes that encode enzymes involved in carbohydrate metabolic process were down-regulated in $S l B S D 1-K D$ leaves.

It is interesting that numerous genes involved in stimulus responses were also up-regulated in SlBSD1-KD leaves. Many of these genes encode chitinase, pathogenesis-related protein, detoxification and heat shock protein, indicating the protective steps which SIBSD1-KDtomatoes takes to respond to the stress generated 
by the degradative and mobilization functions. Significantly, several genes involved in translation were upregulated in SlBSD1-KD leaves, including genes encoding ribosomal protein, ribosome recycling factor and elongation factor. Taken together, our RNA-seq analysis results indicated numerous genes, of which many are tightly related to leaf senescence, are differentially expressed in SlBSD1-KD leaves. These results suggest that, as a transcription factor, SlBSD1 controls expression of large numbers of genes in leaves, including genes involved in leaf senescence.

\section{SIBSD1 and fruit quality}

Delaying leaf senescence and extending the duration of active photosynthesis could substantially increase the instant photoassimilate source and hence increase the grain yield and quality (Ali, Gao, \& Guo, 2018). Sweetness, which results from total soluble sugar, is one of the most important traits of tomato fruits, especially for industrial processing. Given the fact that SIBSD1 plays a negative role in leaf senescence, we next sought to determine whether altered expression of $S I B S D 1$ has effect on fruit sweetness. We measured Brix values of SlBSD1-KD, SlBSD1-OX and the WT tomato fruits. Compared to the WT plants, SlBSD1$K D$ tomatoes showed lower levels (4.61\% decrease) of soluble solids content in ripe fruits, whereas SlBSD1-OX tomatoes showed higher levels (16.25\% increase) of soluble solids content, as manifested by the Brix indexes of the ripe fruits (Fig. 7 ). Collectively, these data suggest that SIBSD1 plays an important role in leaf senescence and fruit quality in tomato.

\section{Discussion}

It has been found that the BSD domain-containing proteins, including transcription factors, exist in a variety of organisms ranging from protozoans to humans. However, not all BSD proteins function as transcription factor, suggesting the BSD domain could possess other conserved functions and be involved more general processes (Doerks et al., 2002). In plants, the first studied BSD protein was Arabidopsis BSD1 (AtBSD1) that is the closest homolog of tomato SlBSD1. AtBSD1 has been characterized as a functional transcription factor localized in the nucleus but its function is unknown (Park et al., 2009). Additionally, banana possesses at least four BSD domain-containing transcription factors namely MaBSD and MaBSD1/2/3, among which MaBSD is involved in fruit ripening, presumably via, at least partially, directly regulating two cell wall modification-related genes, MaEXP1 andMaEXP2 (Ba et al., 2014), whereas MaBSD1, MaBSD2 and MaBSD3 might be involved in cell proliferation during somatic embryogenesis (Maldonado-Borges, KuCauich, \& Escobedo-Graciamedrano, 2013; Shivani et al., 2017). The tomato BSD-containing transcription factor SlBSD1 identified in this study appears to play an important role in vegetative growth and leaf senescence, as manifested by retarded growth and precocious leaf senescence in the SlBSD1-KD plants, in which theSlBSD1 gene is specifically repressed (Fig. 3,4 ). It is notable SlBSD1 and the four banana BSD proteins do not share much similarity and are in different nodes by the phylogenetic analysis (Fig.S5 ), which could explain the difference of their predicted functionalities. In addition, the Arabidopsis T-DNA knockout line of $A t B S D 1$ does not exhibit any growth retardation or early senescence phenotype, which is likely due to functional redundancy of other BSD1 homologs in Arabidopsis (Park et al., 2009). However, this could also suggest BSD transcription factors may have distinct functions in different plant species.

It appears that one of the most important roles of SIBSD1 is controlling vegetative growth and leaf senescence in tomato, which is supported by both loss-of-function and gain-of-function assays. Knockdown ofSIBSD1 in tomato resulted in retarded vegetative growth, whereas overexpression of SlBSD1 rendered the opposite effect (Fig.3 ), suggesting SlBSD1 is essential for plant growth. The genetic analyses also implicate SlBSD1 negatively regulates leaf senescence. The SlBSD1-KD tomato plants, in which theSlBSD1 gene was specifically repressed, displayed precocious leaf senescence, including yellowing of mature green leaves in the 8-week-old plants (Fig. 4a ), accelerated loss of chlorophyll content in the leaves at a later age (7th and 4th leaves, Fig. 4b ), senescence-associated cell death in aged leaflets (7th and 4th leaves, Fig. 4c ), and up-regulation of senescence marker genes (SlSAG12, SlSAG15 , SlSGR1 and SlPPH ) in the third leaf of 8-week-old plants (Fig. 4d ). Significantly, consistent with these observations, the SlBSD1-OX plants overexpressing SlBSD1 exhibited opposite senescence-related phenotypes (Fig. 5 ). In addition, the negative role of SIBSD1 in leaf senescence was also supported by our RNA-Seq analysis on theSIBSD1-KD plants in a 
comparison to the WT plants. We found large number of genes involved in macromolecular degradation are up-regulated, whereas genes involved in chloroplast activity are down-regulated, inSlBSD1-KD leaves. Leaf senescence is a developmental process reflecting aging of leaves but can be triggered precociously by internal cues, such as disruption of homeostasis of phytohormones, and external environmental factors, including abiotic stress and nutrition starvation. Thus, the onset and progression of senescence must be dynamically controlled by the synergistic and/or antagonistic effects of regulatory factors. Several transcription factors have been identified as positive senescence regulators in tomato, including NAC transcription factors SINAP2 (Ma et al., 2018), NOR (Ma et al., 2019), and the orthologs of Arabidopsis ORE1 (SlORE1S01/02/03) (Lira et al., 2017). The identification of the novel transcription SIBSD1 as a negative regulator has now added a new member on the list of leaf senescence regulators in tomato. It is interesting that, like SlBSD1, the NAC transcription factors NOR and SINAP2 also appears possessing pleiotropic functions in plant development, including vegetative growth, age- and dark-induced leaf senescence, and fruit ripening and yield (Ma et al., 2019; Ma et al., 2018).

Positive and negative regulators of leaf senescence could be up-regulated and down-regulated during leaf senescence, respectively. For example, in tomato, both positive regulators NOR andSlNAP1 are up-regulated during leaf senescence (Ma et al., 2019; Ma et al., 2018). Interestingly, the expression of SlBSD1 is not significantly affected during senescence, as indicated by no significant changes of SIBSD1 expression detected by the real-time PCR analysis at different developmental stages: young leaves (YL), mature leaves (ML), senescent leaves (SL), and late senescent leaves (LS). (Fig.S3e ). We speculate that the SlBSD1 transcription factor mainly functions to control the onset and/or progression of senescence during plant growth and development to prevent precocious senescence, which could be critical for plant productivity and quality by avoiding premature aging. However, it is equally possible that SIBSD1 could be down-regulated at the protein level, such as through ubiquitination-mediated degradation, upon the onset of senescence, which awaits further investigation.

Although SlBSD1-KD plants exhibited retarded growth and precocious leaf senescence, it is unlikely that the retarded growth is the consequence of precocious senescence, because precocious leaf senescence did not occur until reaching the 8-week-old stage when growth arrest had already taken place. This phenotype doesn't resemble the one of the tomato slsbpase mutant, in which the growth arrest is likely due to premature leaf senescence occurring in early developmental stage that leads to severe photosynthesis defect-triggered chlorosis, thereby affecting the overall vegetative growth of the plant (Ding, Wang, \& Zhang, 2018).

\section{References}

Ali, A., Gao, X., \& Guo, Y. (2018). Initiation, Progression, and Genetic Manipulation of Leaf Senescence. Methods in molecular biology (Clifton, N.J.), 1744 , 9-31. doi:10.1007/978-1-4939-7672-0_2

Andersson, A., Keskitalo, J., Sjodin, A., Bhalerao, R., Sterky, F., Wissel, K., . . . Nilsson, P. (2004). A transcriptional timetable of autumn senescence. Genome Biol, 5 (4), R24. doi:10.1186/gb-2004-5-4-r24

Arnon, D. I. (1949). COPPER ENZYMES IN ISOLATED CHLOROPLASTS. POLYPHENOLOXIDASE IN BETA VULGARIS. Plant Physiol, 24 (1), 1-15. doi:10.1104/pp.24.1.1

Ba, L. J., Shan, W., Xiao, Y. Y., Chen, J. Y., Lu, W. J., \& Kuang, J. F. (2014). A ripening-induced transcription factor MaBSD1 interacts with promoters of MaEXP1/2 from banana fruit. Plant Cell Rep, 33 (11), 1913-1920. doi:10.1007/s00299-014-1668-6

Balazadeh, S., Kwasniewski, M., Caldana, C., Mehrnia, M., Zanor, M. I., Xue, G. P., \& Mueller-Roeber, B. (2011). ORS1, an H2O2-responsive NAC transcription factor, controls senescence in Arabidopsis thaliana. Molecular plant, 4 (2), 346-360. doi:10.1093/mp/ssq080

Balazadeh, S., Siddiqui, H., Allu, A. D., Matallana-Ramirez, L. P., Caldana, C., Mehrnia, M., . . . MuellerRoeber, B. (2010). A gene regulatory network controlled by the NAC transcription factor ANAC092/AtNAC2/ORE1 during salt-promoted senescence. The Plant journal : for cell and molecular biology, 62 (2), 250-264. doi:10.1111/j.1365-313X.2010.04151.x 
Breeze, E., Harrison, E., McHattie, S., Hughes, L., Hickman, R., Hill, C., . . . Buchanan-Wollaston, V. (2011). High-resolution temporal profiling of transcripts during Arabidopsis leaf senescence reveals a distinct chronology of processes and regulation. The Plant cell, 23 (3), 873-894. doi:10.1105/tpc.111.083345

Buchanan-Wollaston, V. (1997). The molecular biology of leaf senescence. Journal of experimental botany, 48 (2), 181-199. doi:10.1093/jxb/48.2.181

Buchanan-Wollaston, V., Page, T., Harrison, E., Breeze, E., Lim, P. O., Nam, H. G., . . . Leaver, C. J. (2005). Comparative transcriptome analysis reveals significant differences in gene expression and signalling pathways between developmental and dark/starvation-induced senescence in Arabidopsis. The Plant journal : for cell and molecular biology, 42 (4), 567-585. doi:10.1111/j.1365-313X.2005.02399.x

Ding, F., Wang, M., \& Zhang, S. (2018). Sedoheptulose-1,7-Bisphosphatase is Involved in Methyl Jasmonateand Dark-Induced Leaf Senescence in Tomato Plants. International journal of molecular sciences, 19 (11). doi:10.3390/ijms19113673

Doerks, T., Huber, S., Buchner, E., \& Bork, P. (2002). BSD: a novel domain in transcription factors and synapse-associated proteins. Trends in biochemical sciences, 27 (4), 168-170. doi:10.1016/s0968-0004(01)020424

Fernández-Bautista, N., Domínguez-Núñez, J. A., Moreno, M. M. C., \& Berrocal-Lobo, M. (2016). Plant Tissue Trypan Blue Staining During Phytopathogen Infection. Bio-protocol, 6 (24), e2078. doi:10.21769/BioProtoc.2078

Gregersen, P. L., \& Holm, P. B. (2007). Transcriptome analysis of senescence in the flag leaf of wheat (Triticum aestivum L.). Plant biotechnology journal, 5 (1), 192-206. doi:10.1111/j.1467-7652.2006.00232.x

Guo, Y., \& Gan, S. (2005). Leaf senescence: signals, execution, and regulation. Current topics in developmental biology, 71 , 83-112. doi:10.1016/S0070-2153(05)71003-6

Guo, Y., \& Gan, S. (2006). AtNAP, a NAC family transcription factor, has an important role in leaf senescence. The Plant journal : for cell and molecular biology, 46 (4), 601-612. doi:10.1111/j.1365-313X.2006.02723.x

Iben, S., Tschochner, H., Bier, M., Hoogstraten, D., Hozák, P., Egly, J. M., \& Grummt, I. (2002). TFIIH plays an essential role in RNA polymerase I transcription. Cell, 109 (3), 297-306. doi:10.1016/s0092-8674(02)007298

Kim, J. H., Woo, H. R., Kim, J., Lim, P. O., Lee, I. C., Choi, S. H., . . Nam, H. G. (2009). Trifurcate feed-forward regulation of age-dependent cell death involving miR164 in Arabidopsis. Science (New York, N.Y.), 323 (5917), 1053-1057. doi:10.1126/science.1166386

Kim, Y. S., Sakuraba, Y., Han, S. H., Yoo, S. C., \& Paek, N. C. (2013). Mutation of the Arabidopsis NAC016 transcription factor delays leaf senescence. Plant \& cell physiology, 54 (10), 1660-1672. doi:10.1093/pcp/pct113

Li, F., Goto, D. B., Zaratiegui, M., Tang, X., Martienssen, R., \& Cande, W. Z. (2005). Two novel proteins, dos1 and dos2, interact with rik1 to regulate heterochromatic RNA interference and histone modification. Current biology : CB, 15 (16), 1448-1457. doi:10.1016/j.cub.2005.07.021

Lim, P. O., Kim, H. J., \& Nam, H. G. (2007). Leaf senescence.Annual review of plant biology, 58 , 115-136. doi:10.1146/annurev.arplant.57.032905.105316

Lira, B. S., Gramegna, G., Trench, B. A., Alves, F. R. R., Silva, E. M., Silva, G. F. F., . . Rossi, M. (2017). Manipulation of a Senescence-Associated Gene Improves Fleshy Fruit Yield. Plant physiology, 175 (1), 77-91. doi:10.1104/pp.17.00452

Love, M. I., Huber, W., \& Anders, S. (2014). Moderated estimation of fold change and dispersion for RNA-seq data with DESeq2. Genome Biol, 15 (12), 550. doi:10.1186/s13059-014-0550-8

Ma, X., Balazadeh, S., \& Mueller-Roeber, B. (2019). Tomato fruit ripening factor NOR controls leaf senescence. Journal of experimental botany, 70 (10), 2727-2740. doi:10.1093/jxb/erz098 
Ma, X., Zhang, Y., Turečková, V., Xue, G.-P., Fernie, A. R., Mueller-Roeber, B., \& Balazadeh, S. (2018). The NAC Transcription Factor SINAP2 Regulates Leaf Senescence and Fruit Yield in Tomato.Plant physiology, 177 (3), 1286-1302. doi:10.1104/pp.18.00292

Maldonado-Borges, J. I., Ku-Cauich, J. R., \& Escobedo-Graciamedrano, R. M. (2013). Annotation of differentially expressed genes in the somatic embryogenesis of musa and their location in the banana genome.Scientific WorldJournal, 2013 , 535737. doi:10.1155/2013/535737

Matallana-Ramirez, L. P., Rauf, M., Farage-Barhom, S., Dortay, H., Xue, G. P., Dröge-Laser, W., . . . Mueller-Roeber, B. (2013). NAC transcription factor ORE1 and senescence-induced BIFUNCTIONAL NUCLEASE1 (BFN1) constitute a regulatory cascade in Arabidopsis. Molecular plant, 6 (5), 1438-1452. doi:10.1093/mp/sst012

Noodén, L. D. (1988). The Phenomena of Senescence and Aging. In L. D. Noodén \& A. C. Leopold (Eds.), Senescence and Aging in Plants(pp. 1-50). United States: Academic Press.

Park, J., Kim, M. J., Jung, S. J., \& Suh, M. C. (2009). Identification of a Novel Transcription Factor, AtBSD1, Containing a BSD Domain in Arabidopsis thaliana. Journal of Plant Biology, 52 (2), 141-146. doi:10.1007/s12374-009-9015-0

Quirino, B. F., Noh, Y. S., Himelblau, E., \& Amasino, R. M. (2000). Molecular aspects of leaf senescence. Trends in plant science, 5 (7), 278-282. doi:10.1016/s1360-1385(00)01655-1

Reichmuth, C., Becker, S., Benz, M., Debel, K., Reisch, D., Heimbeck, G., . . . Buchner, E. (1995). The sap47 gene of Drosophila melanogaster codes for a novel conserved neuronal protein associated with synaptic terminals. Brain research. Molecular brain research, 32 (1), 45-54. doi:10.1016/0169-328x(95)00058-z

Sessa, G., D'Ascenzo, M., \& Martin, G. B. (2000). Thr38 and Ser198 are Pto autophosphorylation sites required for the AvrPto-Pto-mediated hypersensitive response. Embo j, 19 (10), 2257-2269. doi:10.1093/emboj/19.10.2257

Shivani, Awasthi, P., Sharma, V., Kaur, N., Kaur, N., Pandey, P., \& Tiwari, S. (2017). Genome-wide analysis of transcription factors during somatic embryogenesis in banana (Musa spp.) cv. Grand Naine. PLoS One, 12 (8), e0182242. doi:10.1371/journal.pone.0182242

Soneson, C., Love, M., \& Robinson, M. (2016). Differential analyses for RNA-seq: transcript-level estimates improve gene-level inferences [version 2; peer review: 2 approved]. 4 (1521). doi:10.12688/f1000research.7563.2

Tomato Genome Consortium. (2012). The tomato genome sequence provides insights into fleshy fruit evolution. Nature, 485 (7400), 635-641. doi:10.1038/nature11119

Van Eck, J., Kirk, D. D., \& Walmsley, A. M. (2006). Tomato (Lycopersicum esculentum). Methods Mol Biol, 343 , 459-473. doi:10.1385/1-59745-130-4:459

Wang, Z., Buratowski, S., Svejstrup, J. Q., Feaver, W. J., Wu, X., Kornberg, R. D., . . Friedberg, E. C. (1995). The yeast TFB1 and SSL1 genes, which encode subunits of transcription factor IIH, are required for nucleotide excision repair and RNA polymerase II transcription. Molecular and cellular biology, 15 (4), 2288-2293. doi:10.1128/mcb.15.4.2288

Woo, H. R., Kim, H. J., Lim, P. O., \& Nam, H. G. (2019). Leaf Senescence: Systems and Dynamics Aspects. Annual review of plant biology, 70 , 347-376. doi:10.1146/annurev-arplant-050718-095859

Wu, A., Allu, A. D., Garapati, P., Siddiqui, H., Dortay, H., Zanor, M. I., . . . Balazadeh, S. (2012). JUNGBRUNNEN1, a reactive oxygen species-responsive NAC transcription factor, regulates longevity in Arabidopsis. The Plant cell, 24 (2), 482-506. doi:10.1105/tpc.111.090894

Yang, S. D., Seo, P. J., Yoon, H. K., \& Park, C. M. (2011). The Arabidopsis NAC transcription factor VNI2 integrates abscisic acid signals into leaf senescence via the COR/RD genes. The Plant cell, 23 (6), 2155-2168. doi:10.1105/tpc.111.084913 
Zhang, W. Y., Xu, Y. C., Li, W. L., Yang, L., Yue, X., Zhang, X. S., \& Zhao, X. Y. (2014). Transcriptional analyses of natural leaf senescence in maize. PLoS One, 9 (12), e115617. doi:10.1371/journal.pone.0115617

\begin{tabular}{lll}
\hline Gene class & Num & Example \\
\hline Up-regulated & Up-regulated & Up-regulated \\
Proteolysis & 12 & cysteine proteinase, aspartyl protease, ubiquitin conjugating enzyme, \\
Nucleic acid catabolic process & 1 & ribonuclease \\
Chlorophyll catabolic process & 1 & chlorophyllase \\
Transport & 11 & amino acid transporter, amino acid permease, sugar transporter, ion \\
Response to stimulus & 15 & chitinase, pathogenesis-related protein, detoxification protein, heat sh \\
Down-regulated & Down-regulated & Down-regulated \\
Photosynthesis & 13 & chlorophyll binding protein, photosystem I subunit, photosystem II s \\
Tetrapyrrole biosynthetic process & 5 & protoporphyrinogen oxidase, porphobilinogen deaminase, uroporphyr \\
Carbohydrate metabolic process & 8 & fructose-1,6-bisphosphatase, sucrose synthase, hexokinase 2, glucose-6 6 \\
Translation & 26 & ribosomal protein, elongation factor \\
\hline
\end{tabular}

Table 1 Potential senescence-associated genes showing altered transcript abundance in SlBSD1-KD leaves compared to WT

\section{Figure legends}

Fig. 1. Phylogenetic analysis of BSD domain and BSD domain-containing proteins.

(a) Unrooted tree of BSD domains with the main clades highlighted in different colors. The phylogenetic tree was constructed using the NJ method and a bootstrap test with 1000 replicates. Bootstrap values are marked for the branches that separate the main clades. Names of representative BSDs and locus of tomato BSDs are present at the leaves. Multiple domains in the same protein are labelled a and b.(b) Subtree of BSD domain-containing proteins. The phylogenetic tree was constructed using the NJ method. The numbers listed on each node represent the bootstrap support value associated with that node after running 1000 replicates. (c) SlBSD1 protein (440 amino acids) contains one conserved BSD domain in its center. Black shading indicates the neighboring Phe and Trp residues. Abbreviations: At,Arabidopsis thaliana; Ce, Caenorhabditis elegans ; Dm,Drosophila melanogaster ; Hs, Homo sapiens ; Mm, Mus musculus ; Os, Oryza sativa ; Sc, Saccharomyces cerevisiae; Sl, Solanum lycopersicum; Sp, Schizosaccharomyces pombe .

Fig. 2 SlBSD1 is a functional transcription factor.

(a) Subcellular localization of SIBSD1 in $N$. benthamianaprotoplasts. scale bars, $10 \mu \mathrm{m}$. (b) Transactivation activity of SIBSD1 in yeast, as indicated by blue coloration of yeast cells (transformed with construct of SlBSD1 fused to the DNA binding domain of LexA operator) grown on the selective medium containing X-Gal.(c) qRT-PCR analysis of SlBSD1 transcript in various tomato tissues. The expression level in leaf was defined as "1". Data are mean $\pm 95 \%$ CL $(n=3)$.

Fig. 3 SlBSD1 plays in important role in vegetative growth in tomato.

(a) 6-week-old SlBSD1 knockdown (SlBSD1-KD),SlBSD1 overexpression (SlBSD1-OX ) and wild type (WT) plants. Scale bar, $10 \mathrm{~cm}$. (b) Plant height of SlBSD1 knockdown (SlBSD1-KD), SlBSD1 overexpression (SlBSD1-OX ) and wild type (WT) plants at 4-week, 6-week and 13-week stage.

Fig. $4 S l B S D 1$ knockdown $(S l B S D 1-K D)$ plants display an early senescence phenotype.

(a) Phenotype of WT and SlBSD1-KD plants. Upper panel, 8-week-old whole plants, scale bars, $10 \mathrm{~cm}$; lower panel, detached leaflets from the $3^{\text {rd }}$ true leaf of 8 -week-old plants, scale bars, $1 \mathrm{~cm}$. (b) Total chlorophyll content of the $10^{\text {th }}, 7^{\text {th }}$ and $4^{\text {th }}$ true leaves of 8 -week-old WT and SlBSD1-KD plants. FW, fresh weight. (c) Trypan blue staining of detached leaflets from the $10^{\text {th }}, 7^{\text {th }}$ and $4^{\text {th }}$ true leaves of 8-week-old WT and SlBSD1$K D$ plants. Blue-colored patches indicate areas of dead or dying cells. Scale bars, $1 \mathrm{~cm}$.(d) Expression level 
of senescence marker gene SlSAG12 ,SlSAG15, SlSGR1 and SlPPH in the $3^{\text {rd }}$ true leaves of 8-week-old WT and SlBSD1-KD plants. Data presented In (b) and (d) were means \pm SD $(\mathrm{n}=3)$. Asterisks indicated statistically significant difference compared with WT. ${ }^{*} P<0.05,{ }^{* *} P<0.01$; two-tailed Student's $t$-test.

Fig. 5 Developmental leaf senescence is delayed in SlBSD1 overexpression (SlBSD1-OX ) plants.

(a) Phenotype of WT and SlBSD1-OX plants. Upper panel, 12-week-old whole plants, scale bars, $10 \mathrm{~cm}$; lower panel, detached leaflets from the $3^{\text {rd }}$ true leaf of 12 -week-old plants, scale bars, $1 \mathrm{~cm}$. (b) Total chlorophyll content of the $10^{\text {th }}, 7^{\text {th }}$ and $4^{\text {th }}$ true leaves of 12 -week-old WT and SlBSD1-OX plants. FW, fresh weight. (c) Trypan blue staining of detached leaflets from the $10^{\text {th }}, 7^{\text {th }}$ and $4^{\text {th }}$ true leaves of 12 -week-old WT and SlBSD1-OX plants. Blue-colored patches indicate areas of dead or dying cells. Scale bars, $1 \mathrm{~cm} .(\mathrm{d})$ Expression level of senescence marker gene SlSAG12,SlSAG15, SlSGR1 and SlPPH in the $3^{\text {rd }}$ true leaves of 12-week-old WT and SlBSD1-OX plants. Data presented In (b) and (d) were means $\pm \mathrm{SD}(\mathrm{n}=3)$. Asterisks indicated statistically significant difference compared with WT. ${ }^{*} P<0.05,{ }^{* *} P<0.01$; two-tailed Student's $t$-test.

Fig. 6 SlBSD1 control dark-induced senescence.

(a) Young detached leaflets of 6-week-old plants as indicated before (0 DDI) and after 10 days of dark incubation (10 DDI). Scale bars, $1 \mathrm{~cm}$. (b) Trypan blue staining of leaflets after 10 days of dark incubation. Blue-colored patches indicate areas of dead or dying cells. Scale bars, $1 \mathrm{~cm}$. (c) Total chlorophyll content in the leaflets before and after 10 days of dark incubation. FW, fresh weight.(d) Expression level of senescence marker genes SlSAG12,SlSAG15, SlSGR1 and SlPPH in the leaflets after 10 days of dark incubation. Data presented In (b) and (c)were means $\pm \mathrm{SD}(\mathrm{n}=3)$. Asterisks indicated statistically significant difference compared with WT. ${ }^{*} P<0.05,{ }^{*} P<0.01$; two-tailed Student's $t$-test.

Fig. 7 Soluble solids content in SlBSD1 knockdown (SlBSD1-KD), SlBSD1 overexpression (SlBSD1-OX ) and wild type (WT) ripe fruits. 
(a)

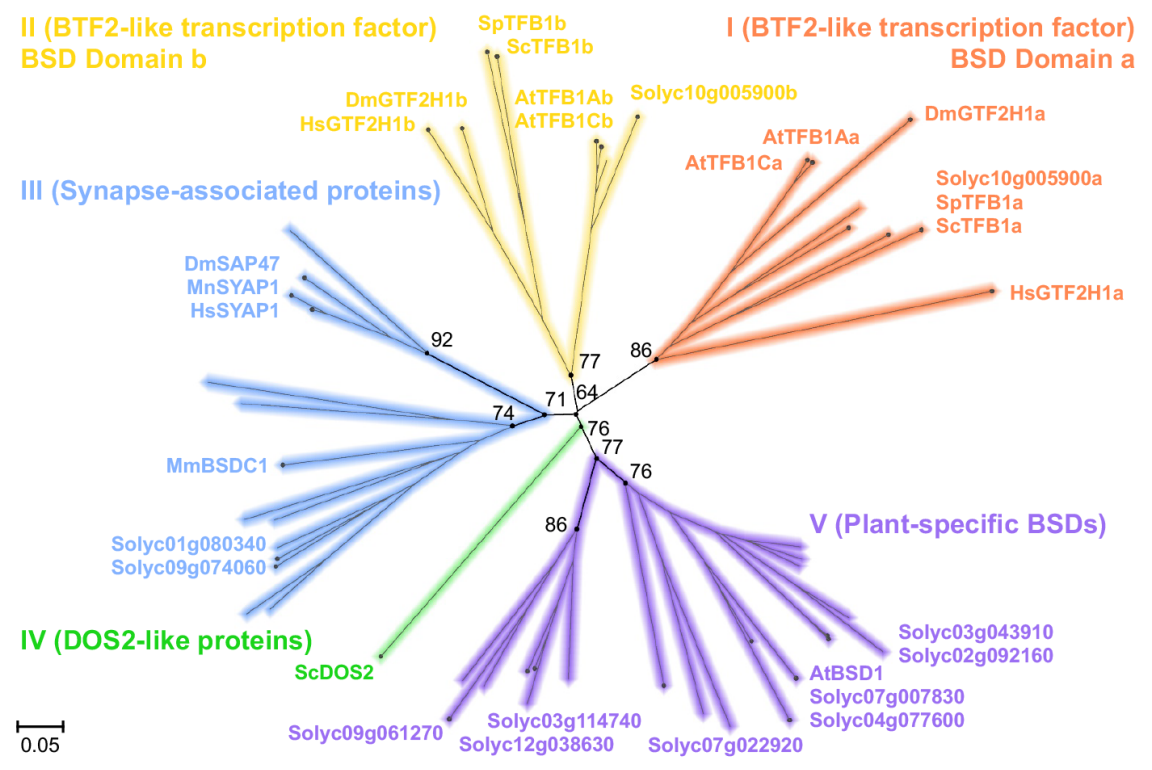

(b)

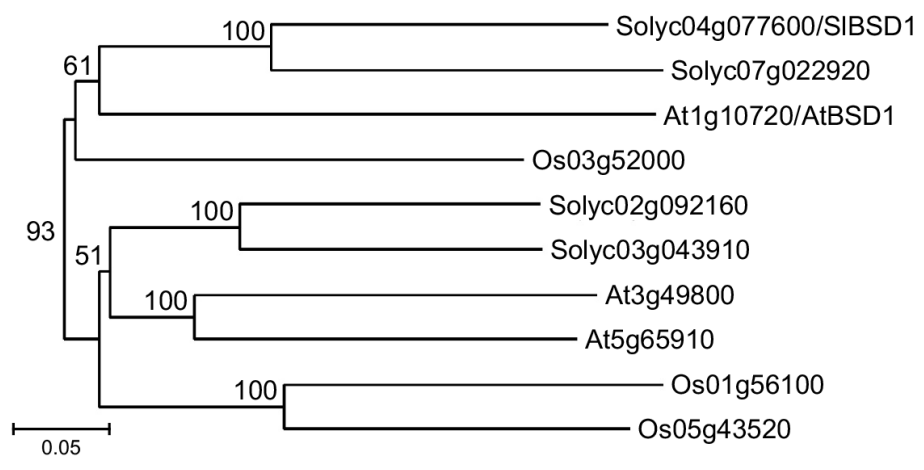

(c)

\begin{tabular}{|r|r|r|}
\hline & \multicolumn{2}{c}{254} \\
\hline & BSD & \\
\hline
\end{tabular}



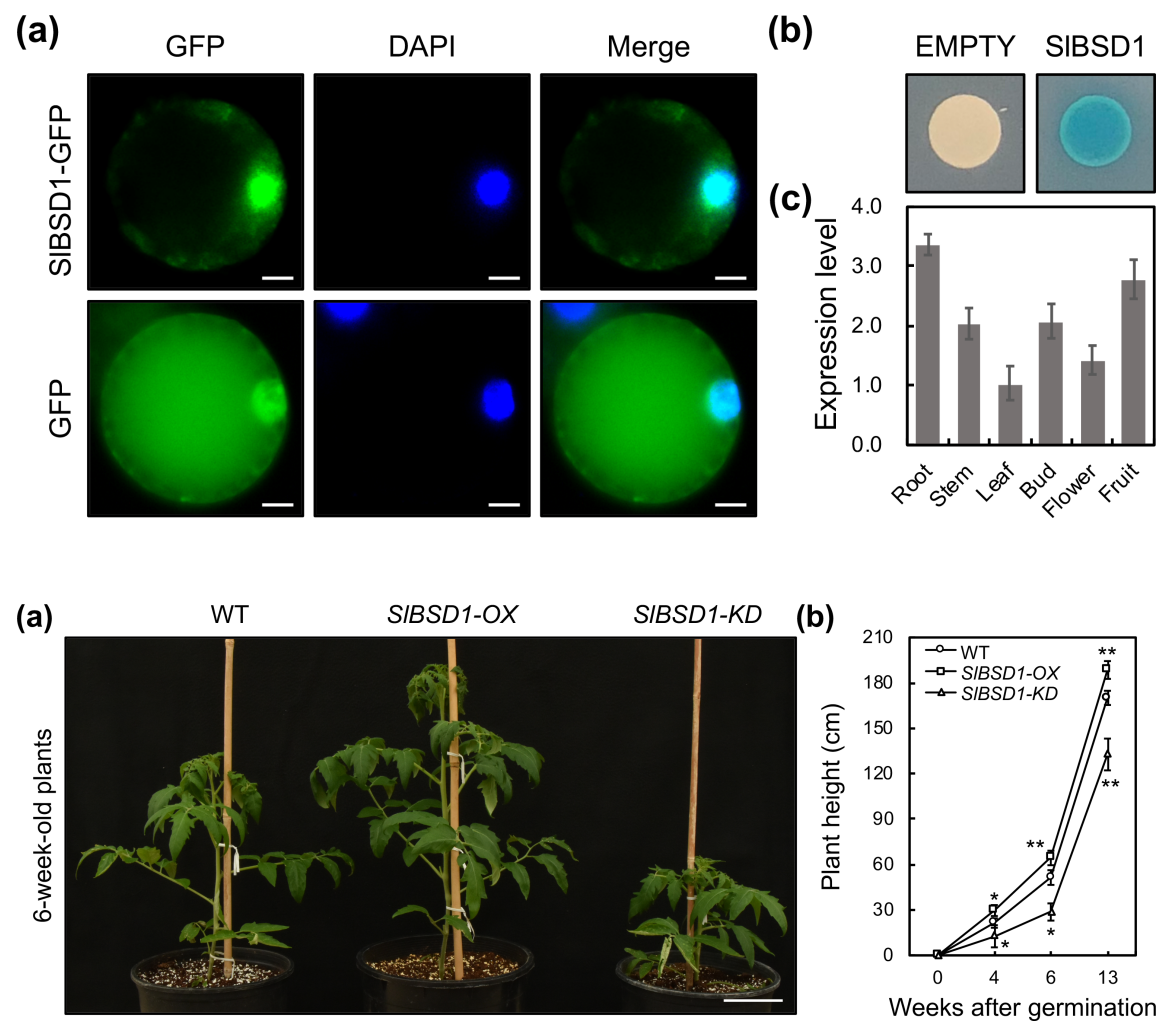


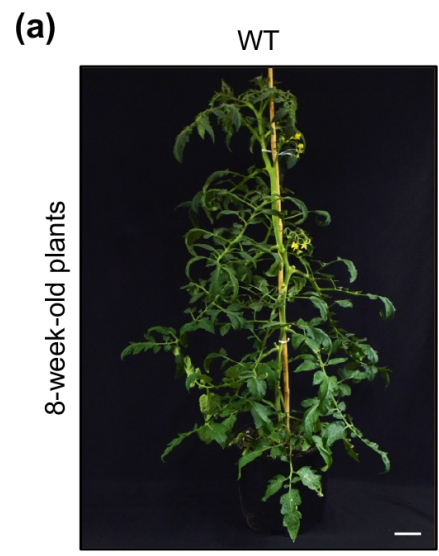

SIBSD1-KD

(b)
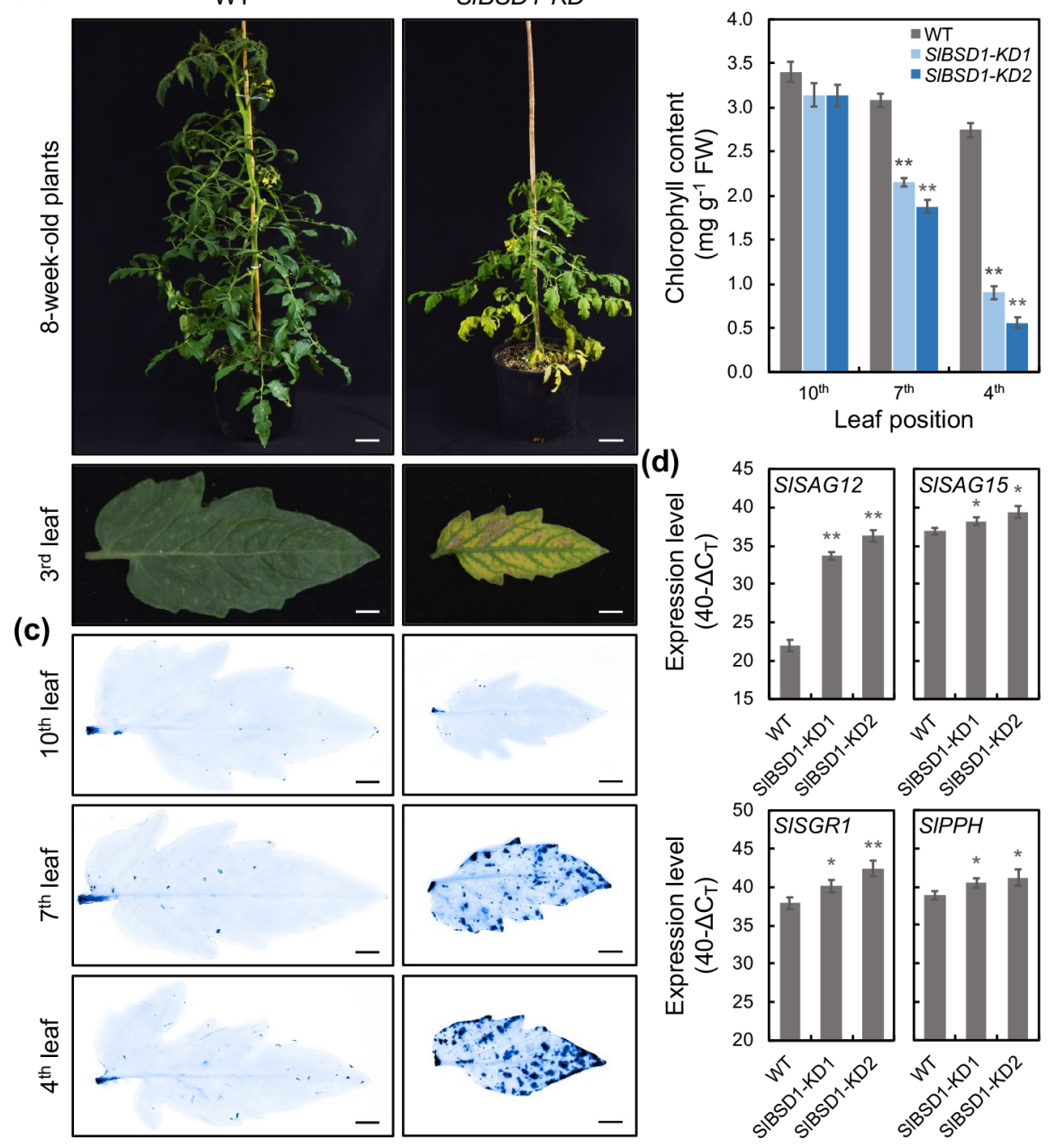


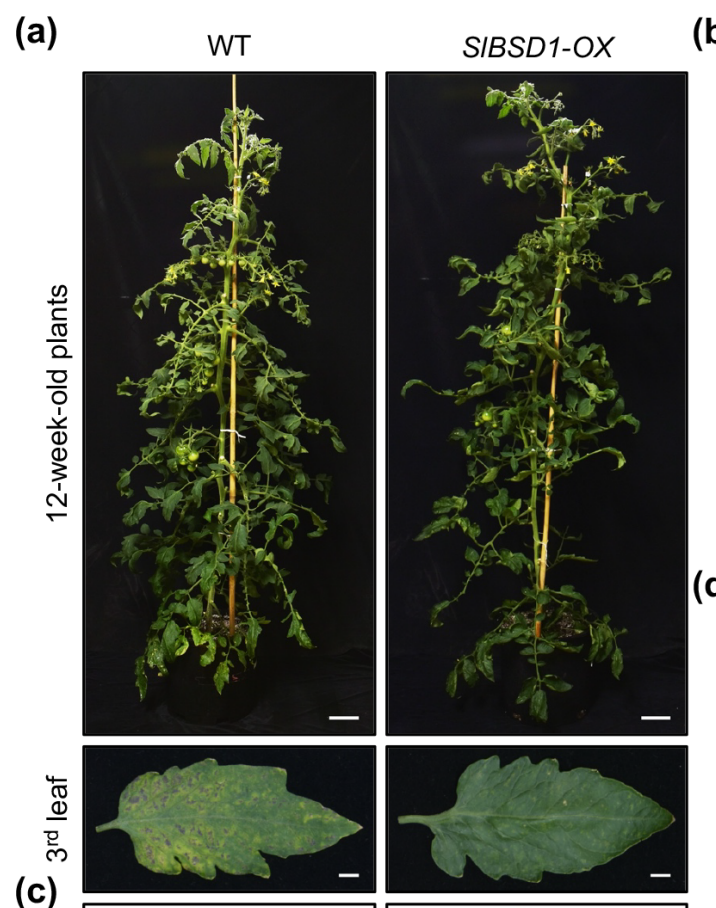

(b)
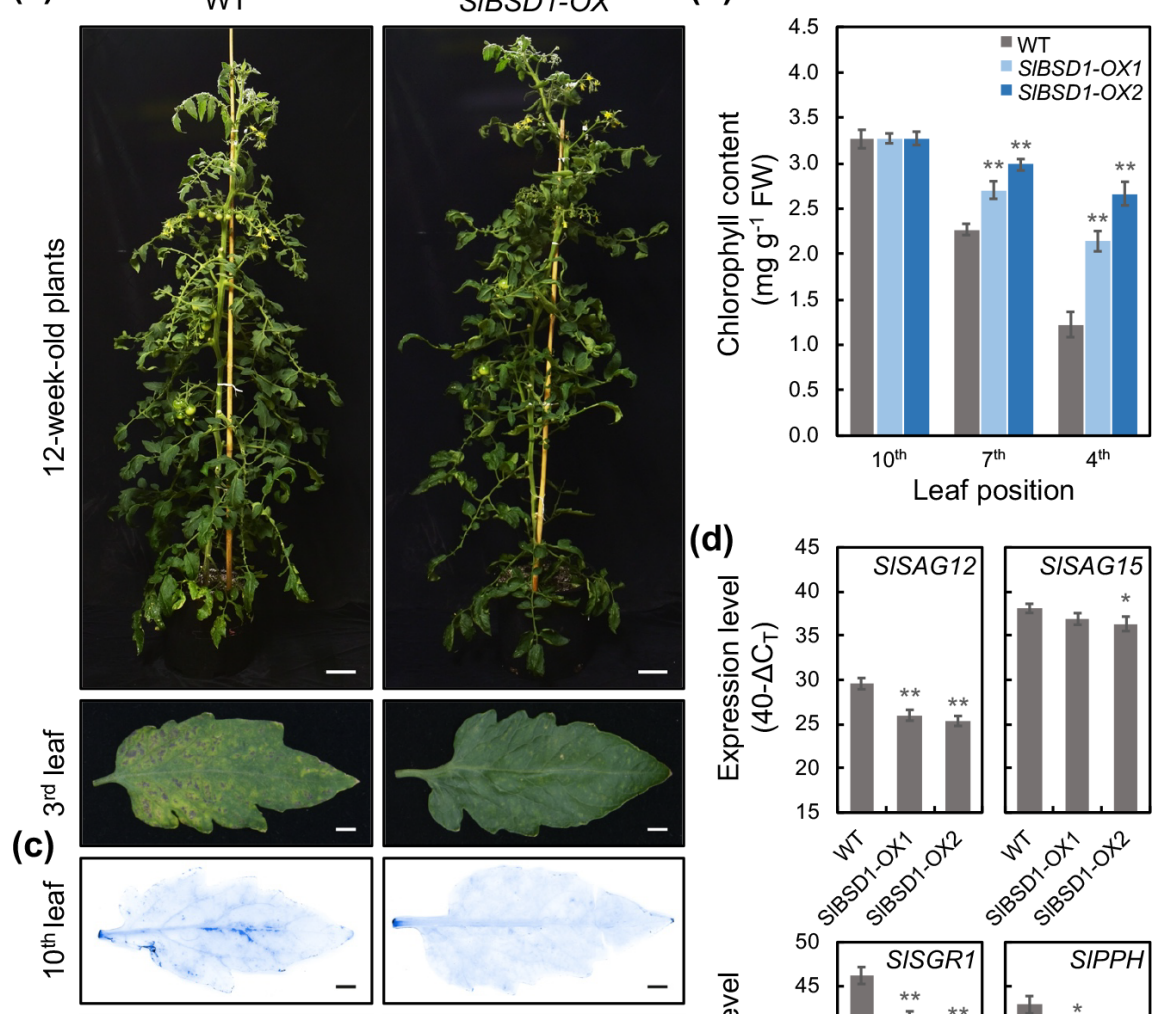

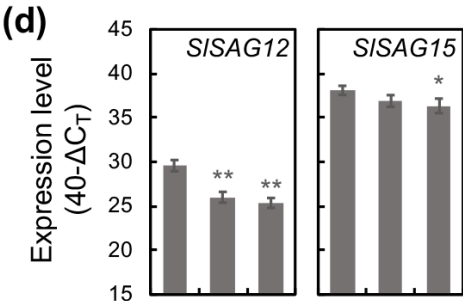

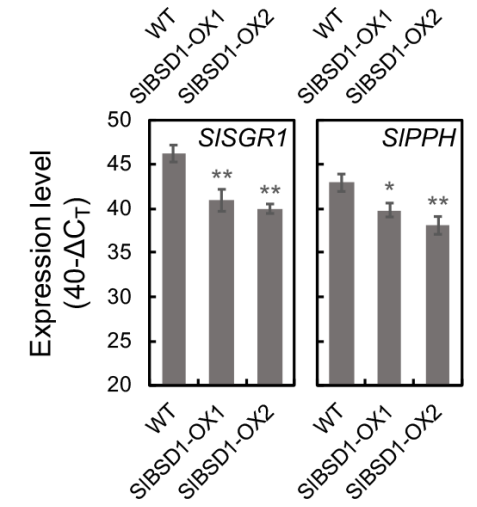


(a)
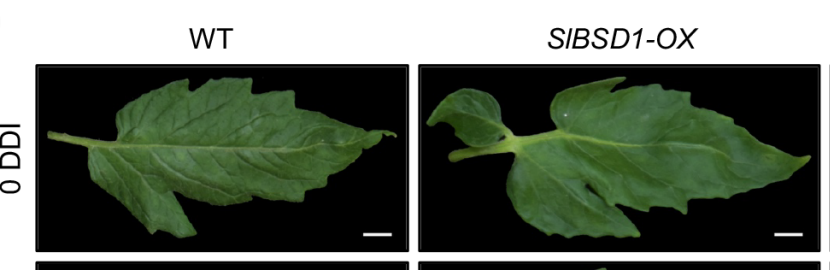

SIBSD1-KD

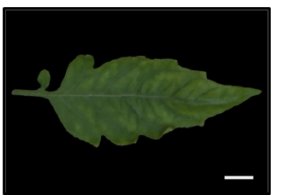

(b)
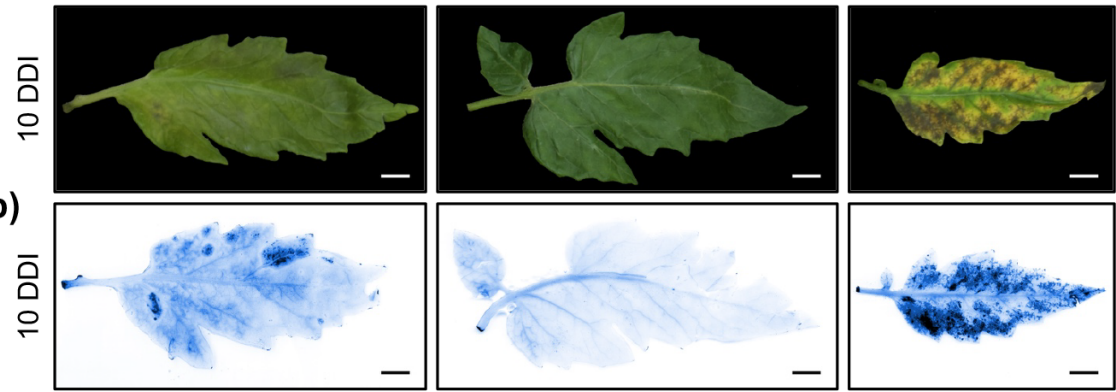

(c)

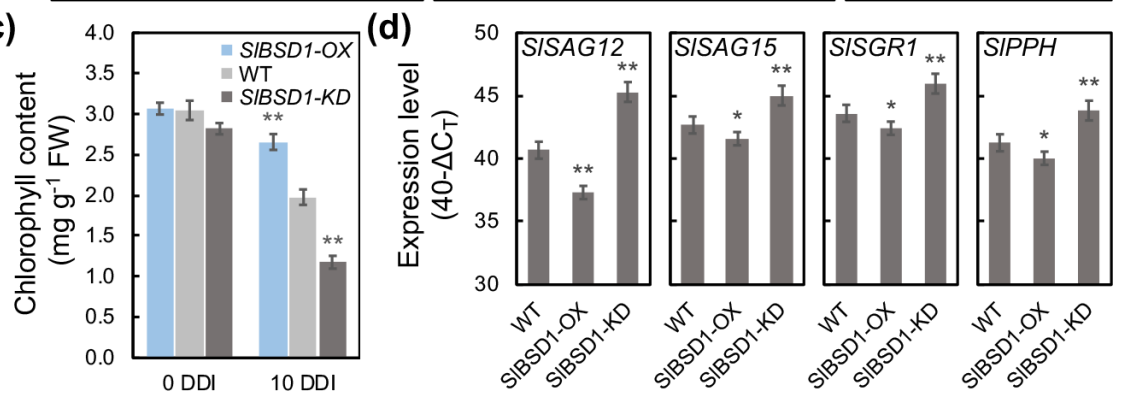




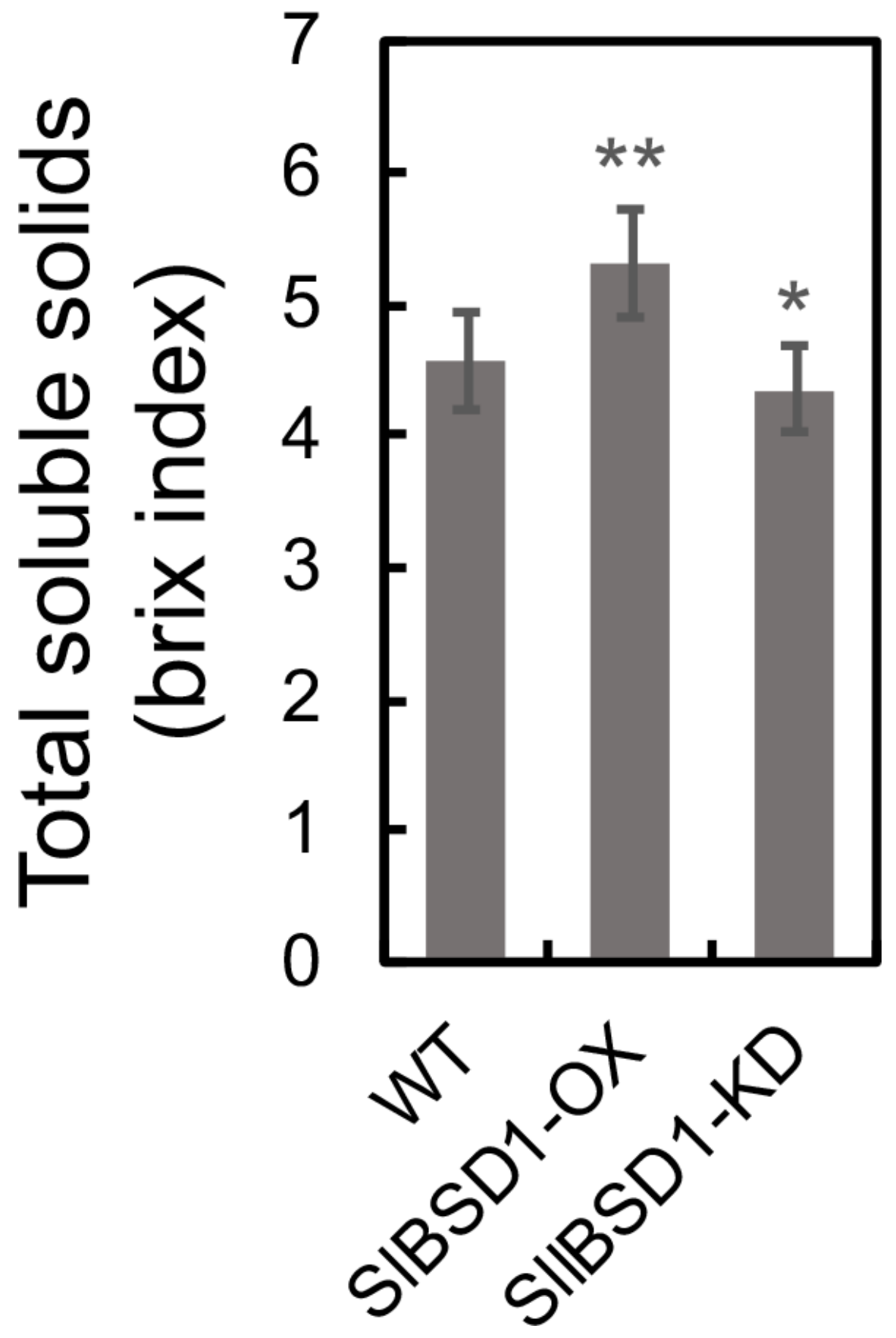

